Proceedings of the $\mathbf{2}^{\text {nd }}$ ICEENG Conference, 23-25 Nov. 1999

\begin{tabular}{|l|l|}
\hline CS-1 & 1 \\
\hline
\end{tabular}

Military Technical College

Kobry Elkobbah, Cairo, Egypt

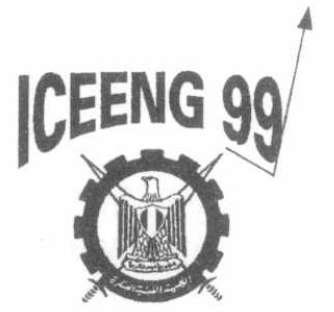

$2^{\text {nd }}$ International Conference on Electrical Engineering ICEENG 99

\title{
A PROPOSED METHOD FOR IMPROVING THE PERFORMANC OF P-TYPE InP IMPATT
}

\author{
Hosny A. El-Motaafy*
}

\section{ABSTRACT}

A special waveform is proposed and assumed to be the optimum waveform for p-type InP IMPATTS. This waveform is deduced after careful and extensive study of the performance of these devices. The results presented here indicate the superiority of the performance of the IMPATTs driven by the proposed waveform over that obtained when the same IMPATTs are driven by the conventional sinusoidal waveform. The results presented here are obtained using a full-scale computer simulation program that takes fully into account all the physical effects pertinent to IMPATT operation. In this paper, it is indicated that the superiority of the proposed waveform is attributed to its ability to reduce the bad effects that usually degrade the IMPATT performance such as the space-charge effect and the drift-velocity dropping below saturation effect. The superiority is also attributed to the ability of the proposed waveform to improve the phase relationship between the terminal voltage and the induced current.

\section{KEY WORDS}

IMPATT diode, In P, Special waveforms, Avalanche process, Space-charge effect, Drift velocity, Velocity-field characteristic, Drift-velocity dropping below saturation.

* Dept. of Electronics and Computer Engineering, Higher Tech. Institute, $10^{\text {th }}$ of Ramadan City 


\section{INTRODUCTION}

It is well known that the performance of n-type InP IMPATT diodes is superior to that of $p$-type diodes [1-4]. This is attributed mainly to the velocity-field characteristic of electrons in the InP material that has a negative-differential mobility region [1-4]. This allows the electrons to have higher velocities at the lower values of field encountered in the active region during the negative half cycle of operation [2-4]. This is in contrast to holes whose velocity drops below the saturated drift velocity for the lower values of the field. Since these effects are inherent in InP material, it is only possible to improve the performance of p-type InP IMPATTs through modifying the microwave circuit where the diode is imbedded. Since it has been previously reported that the performance of IMPATTs could be improved if it is driven by a nonsinusoidal voltage waveform [ 4-7 ], the external circuit attached to the diode must be capable of supporting such waveforms. However, no comprehensive study has been conducted for determining the optimum waveform. In this paper, a systematic procedure for choosing the optimum waveform and designing the external microwave circuit is presented. A special waveform is proposed to be the optimum waveform for p-type InP IMPATT. This proposition is supported by an extensive investigation of IMPATT operation [1-3, 7-8]. This investigation has been conducted using a modified version of the full-scale computer simulation program described elsewhere [8]. This program takes fully into account all the physical effects pertinent to IMPATT operation without resorting to approximations that limit the scope of its application.

The performance of a given IMPATT that is expected to be modest is studied when it is driven by this waveform and when it is driven by the conventional sinusoidal waveform. Then the results obtained for the two cases are compared. The superiority of the proposed waveform, (PWF) is demonstrated specially at higher values of the dc current density $J_{d c}$. The PWF can be optimized further through optimizing its parameters. Since the PWF is difficult to be realized practically, the performance of the IMPATT is studied when it is driven by the waveform V2. This waveform consists of the first two harmonics of the proposed waveform. The performance in this case is found also to be much more superior to the conventional case.

\section{THE PROPOSED WAVEFORM}

The proposed waveform (VF) is shown in Fig. 1 for the case where $A_{2}=90 \mathrm{~V}$. In the first part of the cycle, $\Phi_{1}$ the height of the PWF is $A_{1}$. In the second part $\Phi_{2}$, the height is $A_{2}$. In the negative portion of the cycle, the height is $A_{3}$. The transitions between the different regions are $\varepsilon_{1}, \varepsilon_{2}$ and $\varepsilon_{3}$ respectively. The waveform consisting of the first two harmonics of the proposed waveform denoted by V2 is shown in the same Fig. $1 \mathrm{~b}$ together with the conventional waveform (VC). The parameters of this waveform can be optimized according to the following guidelines that are deduced from our extensive study of IMPATT operation $[1-3,7,8]$ :

1. $\varepsilon_{1}$ should be as small as possible to reduce the spread of transit times of charge carriers. 
2. $\varepsilon_{2}$ should be as small as possible to increase the slope of the terminal voltage when crossing the breakdown level. This helps reduce the space charge effect. 3. $A_{1}$ should be equal to zero to reduce the power dissipation at the beginning of the RF cycle.

4. The choice of $\Phi_{2}$ and $A_{2}$ is a compromise between the following factors :

a. $\Phi_{2}$ should be minimized in order to reduce the spread of transit times of carriers.

b. $A_{2}$ must be increased to reduce the space charge effect and the spread of transit times of carriers and to satisfy the breakdown condition. However, $A_{2}$ must not be too high to avoid the excessive increase of the dc-breakdown voltage. This is attributed to the saturation of the ionization rates at the high values of electric field [ $4,8]$.

c. The product $\Phi_{2} A_{2}$ must be as high as possible to enable the increase of $A_{3}$ which improves the performance.

5. The choice of $A_{3}$ and $\Phi_{3}$ is a compromise between the following factors :

a. The magnitude of $A_{3}$ must be sufficiently high in order to extract the maximum energy from the drifting carriers but must not be too high to cause the drift velocity of carriers to drop below the saturated value.

b. $\Phi_{3}$ must be reduced to delay the injection of the avalanche-generated packet in the drift region but if is excessively reduced the conductance of the diode may become positive at some higher order harmonics.

\section{THE RESULTS OBTAINED FOR THE CHOSEN IMPATT}

The IMPATT whose performance will be studied is an In P p-type one having a single-drift abrupt junction structure. The doping density in the drift region is $3 \times 10^{15}$ $\mathrm{cm}^{-3}$ and its width is $3 \mu \mathrm{m}$.

The PWF has the following parameters :

$\varepsilon_{1}=\varepsilon_{2}=\varepsilon_{3}=9$ degrees, $\Phi_{1}=72^{\circ}, \Phi_{2}=90^{\circ}, \Phi_{3}=171^{\circ}$,

$A_{2}=90 \mathrm{~V}$, and $A_{3}=49.5 \mathrm{~V}$.

Figs. 2 and 3 show the efficiency and the RF power density versus the dc current density $\mathrm{J}_{\mathrm{dc}}$ for the PWF, VF, and a sinusoidal signal of amplitude $70 \mathrm{~V}$. The value of $70 \mathrm{~V}$ is chosen since it was found during the investigation to give the best performance for the sinusoidal excitation at the highest values of $J_{\mathrm{dc}}$ considered. The operating frequency is $15 \mathrm{GHz}$. The results indicate clearly the superiority of the proposed waveforms over the conventional sinusoidal signal especially at the higher values of the dc bias current. This demonstrates the ability of the proposed 
waveforms to reduce considerably the space-charge effect. This is illustrated clearly in Fig. 4 which shows the induced current $J_{i}$ for the proposed waveform and the sinusoidal signal at $\mathrm{J}_{\mathrm{dc}}=5000 \mathrm{~A} / \mathrm{CM}^{2}$. It is clear that the major manifestation of the reduction of the space charge effect is the delay of the injection of the avalanche-generated packet in the drift region. This means that the time delay provided by the avalanche process is increased. This contributes to the improvement of the performance. On the other hand the shape and the values of the induced current in the negative-half cycle are better for the proposed waveforms. This is attributed to the reduction of the spread of transit times of carriers for the PWFs. This is illustrated clearly in Fig.5 that shows the induced current at the dc bias current of $5 \mathrm{KA} / \mathrm{cm}^{2}$. The reduction of the spread of transit times of carriers means that the generated pulse of holes will be more localized in both the time and the space domains. This is clearly demonstrated in Figs. 5 and 6 that show the spatial distribution of the hole current density $\left(J_{p}\right)$ for the considered waveforms together with the electric field $(E)$ at different phase angles $(O)$.

At $\mathrm{O}=90 \mathrm{deg}$., $\mathrm{E}$ is maximum alongside the diode. The avalanche generation process has already started for VC whereas no carriers are generated for VF.

At $\mathrm{O}=162 \mathrm{deg}$., the avalanche-generated packet of holes (AGP) is already formed and injected into the drift region (DR) for VC. After this phase angle, the space charge effect causes $E$ to be depressed behind the AGP. Consequently, the avalanche generation is shut down early in the cycle for VC. Hence, $J_{i}$ has its maximum value at $162 \mathrm{deg}$. At $\mathrm{O}=171 \mathrm{deg}$., $\mathrm{E}$ is so high for VF that the avalanche generation is not affected by the space charge effect of the generated carriers.

At $\mathrm{O}=180 \mathrm{deg}$., the avalanche generation is shut down completely for VF. Consequently, the spread of transit times of charge carriers is reduced. This improves the performance. At this phase angle, the peak of the AGP is at the diode distance $X=1.9 \mu \mathrm{m}$ for VC whereas the peak of the AGP is still at $X=1.1 \mu \mathrm{m}$ for VF. For this waveform, the AGP arrives at $X=1.9 \mu \mathrm{m}$ only at $O=225 \mathrm{deg}$. as Fig. $6 \mathrm{~d}$ shows. The extraction of the AGP occurs earlier for VC. For this waveform, the major portion of the AGP has been already extracted at $O=270 \mathrm{deg}$. At this phase angle, the peak of the AGP is still in the DR for VF. This explains why the induced current $\mathrm{J}_{i}$ ) is higher for VF in the negative portion of the cycle. This higher induced current means that the performance of VF is better than that of VC.

At the end of the cycle, the number of carriers remaining in the DR is higher for VF. These carriers have to be extracted at the beginning of the next RF cycle. However, since the RF voltage is equal to zero during this portion of the cycle for VF, there will be no power dissipation during the carrier extraction.

Fig. 7 show the results obtained for $V F$ and $V C$ at $J_{d c}=2 \mathrm{KA} / \mathrm{cm}^{2}$. It is seen that the AGP is slightly sharper for VF. The peak of $J_{1}$ is also sharper for VF. This means that the spread of transit times is smaller for VF and its AGP is more localized in both the space and time domains. This contributes to the better performance of VF.

Briefly, the avalanche breakdown is considerably delayed for the proposed waveforms. This contributes to their better performance since the phase delay provided by the avalanche process is enhanced. The injection of the avalanchegenerated packet (AGP) is also delayed for the proposed waveform. Correspondingly, the phase delay is increased. This effect improves considerably the performance. As the AGP drifts toward the ohmic contact it is dispersed by the effect of diffusion. This dispersion is enhanced by the space-charge effect for VC. 
For VF, the dispersion is smaller. This is attributed to the facts that the AGP is sharper in both the space and the time domains and the space-charge effect is less significant. The reduction of the spread of transit times means that the currents induced by the carriers have a better distribution of phase angles. Consequently, the induced current will be higher. The drift-velocity dropping below saturation is less significant for VF. This causes almost all the holes to drift at the saturated velocity. Hence, the induced current is higher for this waveform. This contributes to its better performance.

When the diode is driven by V2 it gives a performance that is almost similar to VF. At $J_{d c}=5 \mathrm{KA} / \mathrm{cm}^{2}$, the efficiency is $16.1 \%$ against $18.3 \%$ for VF and $7.6 \%$ for VC. Since, the microwave circuit necessary to support V2 is considerably simpler than that of VF, V2 can be considered the optimum waveform for $p$-type in P IMPATT diodes.

\section{THE REASONS LEADING TO THE SUPERIORITY OF THE PROPOSED WAVEFORMS}

The superiority of the performance of the IMPATTs driven by the proposed waveforms is attributed to the following reasons :

1. The space charge effect is considerably reduced. This is attributed to the high value of the slope of the terminal voltage when crossing the breakdown level and to the large value of this voltage in the time interval during which the avalanche breakdown takes place.

2. The generation of minority carriers in the drift region is diminished. This is attributed to the reduction of the space charge effect.

3. The phase delay provided by the avalanche process is increased. This is because both the start of this process and the injection of the AGP in the drift region are delayed. This is attributed to the favorable shape of the proposed waveform and its ability to reduce both the space-charge effect and the minority-carrier enhancement effect. Therefore, the phase relationship between the terminal voltage and the induced current is improved.

4. The spread of transit times of carriers is reduced. This is also attributed to the shape of the proposed waveform that helps reduce both the space charge effect and the minority carrier enhancement effect. The proposed waveform causes the carrier generation by the avalanche multiplication to be localized in the time domain.

5. Both the drift-velocity dropping below saturation and the depletion-layer width modulation are reduced. This is because of the higher optimum values of the operating dc bias current.

\section{CONCLUSIONS}

In this paper, the performance of the p-type IMPATT is studied when it is driven by a proposed non-sinusoidal waveform. It is indicated that using this waveform, the bad effects that usually degrade the IMPATT performance are reduced. These bad effects include the space-charge effect, the drift-velocity dropping below saturation effect, and the minority-carrier enhancement effect. It is also illustrated that the proposed waveform helps increase the phase delay provided by the avalanche process and reduce the spread of transit times of carriers. All these factors 
contribute to the superiority of the proposed waveform over the conventional one. The performance is also studied when the p-type IMPATT is driven by waveforms consisting only of the first two or three harmonics of the proposed waveform. In this case, the performance of the IMPATT is found to be superior to that obtained when the device is driven by the proposed waveform.

\section{REFERENCES}

[1] El-Motaafy, $\mathrm{H}$. et Al.,"Analysis of Low-High-Low InP IMPATT reflection amplifiers",ANTEM'98, Symposium on Antenna and applied electromagnetics, Ottawa, Ontario, Canada, Aug. (1998).

[2] El-Motaafy, H. et al., "Analysis and Design considerations of a proposed LowHigh-Low InP Distributed IMPATT diode", ICM,97, The International Conference on Microelectronics, Pandung, Indonesia, Oct. (1997).

[3] El-Motaafy, $\mathrm{H}$. et al., "Effect of the variation of the doping density on the performance of low-high-low InP IMPATT diodes ", 1977 North American Radio Science Meeting (IEEE AP-s, International Symposium and URSI Radio-science Meeting, Montrial, Canada, July, (1997).

[4] Bauhahn, P. and Haddad, J. I., " IMPATT device simulation and properties," IEEE Trans. Electron Devices, vol. ED-24, Jan., (1977).

[5] Mouthan, K. "Two frequency operation of the avalanche-transit-time oscillators, " Proc. IEEE(Lett.), vol. 58, March, (1970).

[6] Schroeder, W. E. and Haddad, G. I., " Effect of harmonic and subharmonic signals on avalanche diode oscillator performance, "IEEE Trans. Microwave Theory Tech., vol.MTT-18, June, (1970).

[7] El-Motaafy, H., " The optimum voltage for Si-IMPATT Diodes," Seventh Conference on Solid-State Science, Cairo, (1984).

[8] El-Motaafy, H., "Full-scale computer simulation of TRAPATT diodes, " The Third National Radio-Science Symposium, Cairo, (1985). 

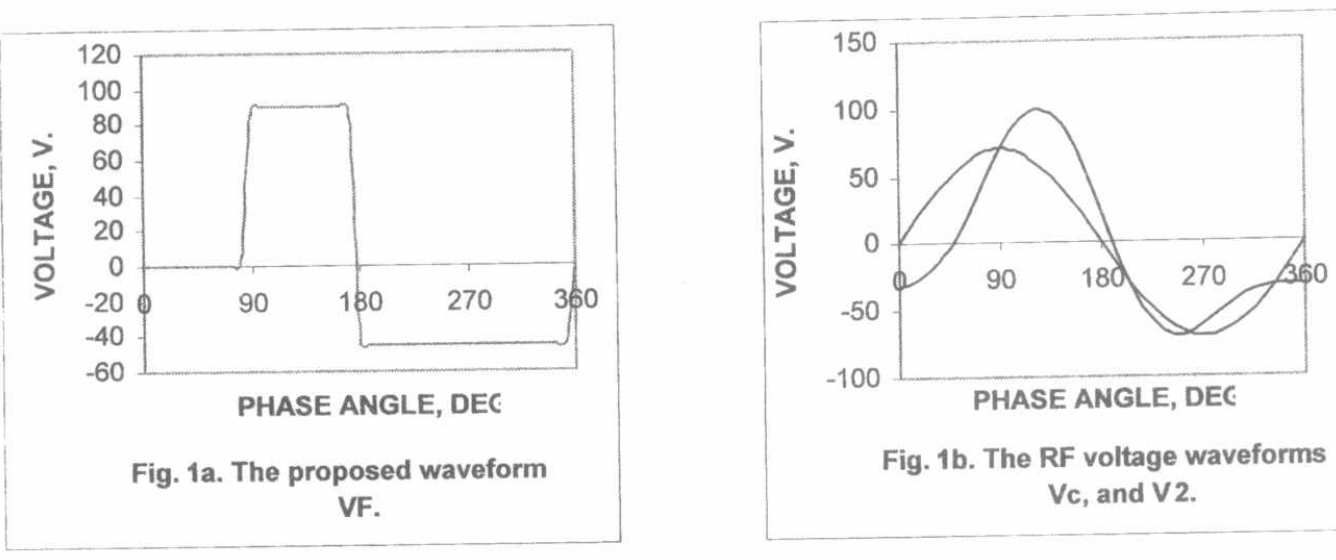

Fig. 1b. The RF voltage waveforms Vc, and V2.
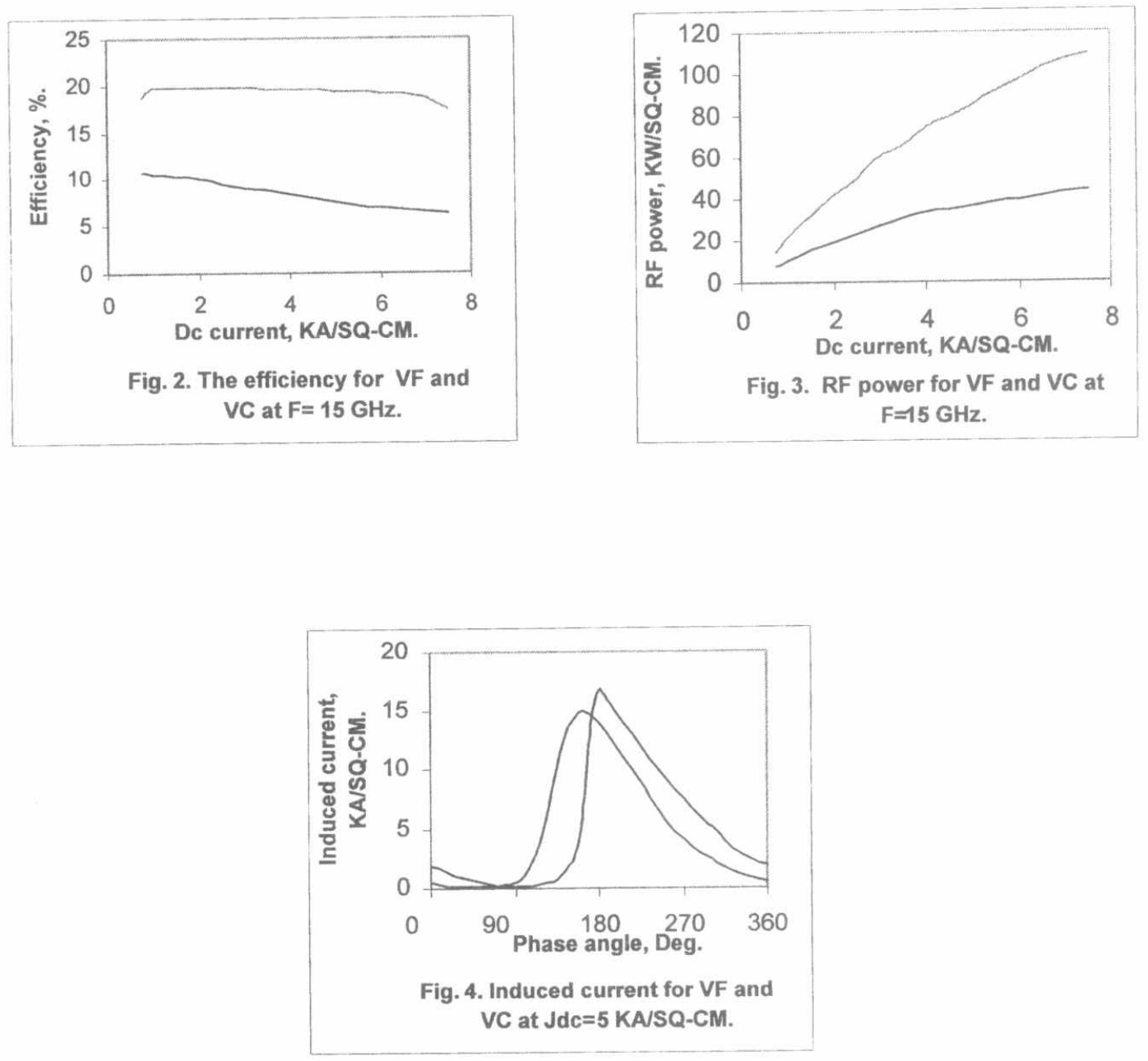


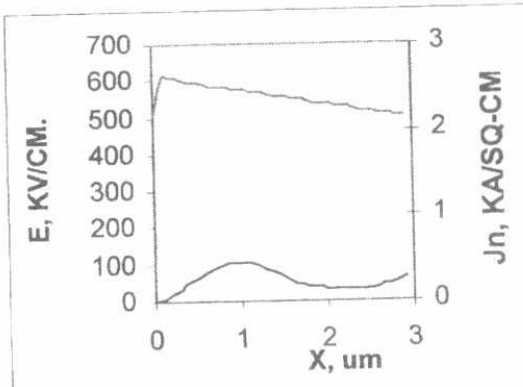

Fig. 5a. Electric field and hole current at $O=90$ deg.

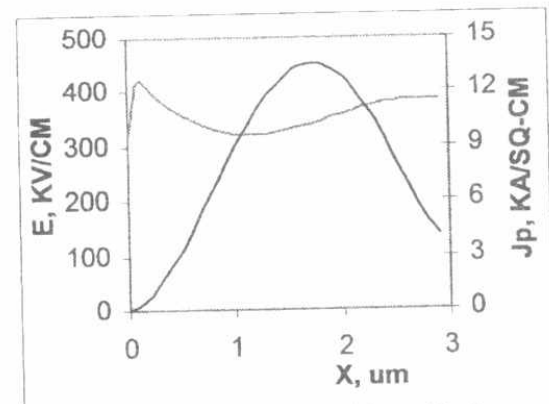

Fig. 5c. Electric field and hole current at $O=171 \mathrm{deg}$.

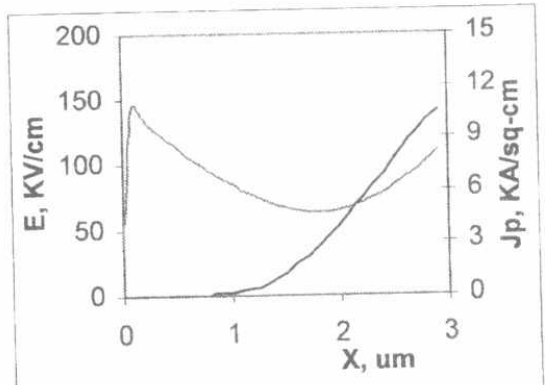

Fig. 5e. Electric field and hole current at $O=270 \mathrm{deg}$.

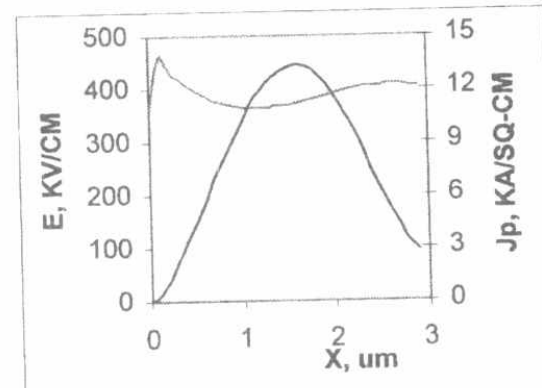

Fig. 5b. Electric field and hole current at $O=162 \mathrm{deg}$.

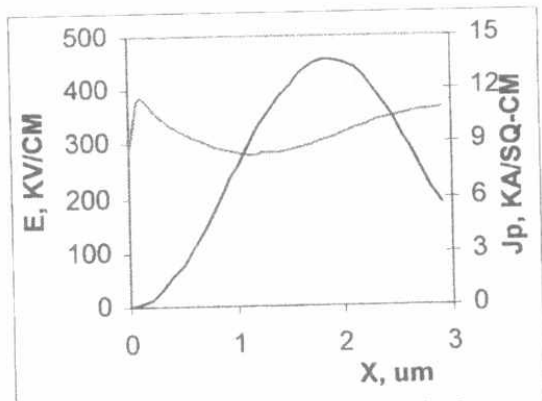

Fig. 5d. Electric field and hole current at $O=180 \mathrm{deg}$.

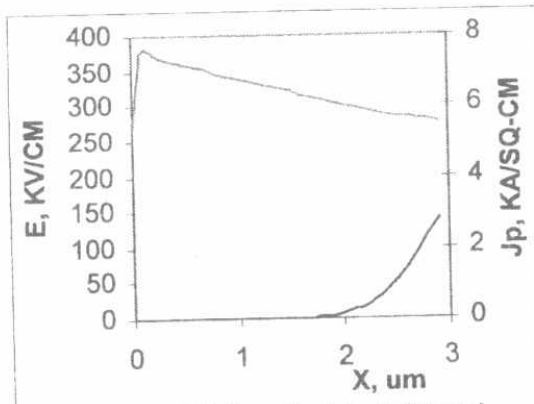

Fig. 5f. The electric field and the hole current at $O=360 \mathrm{deg}$. 

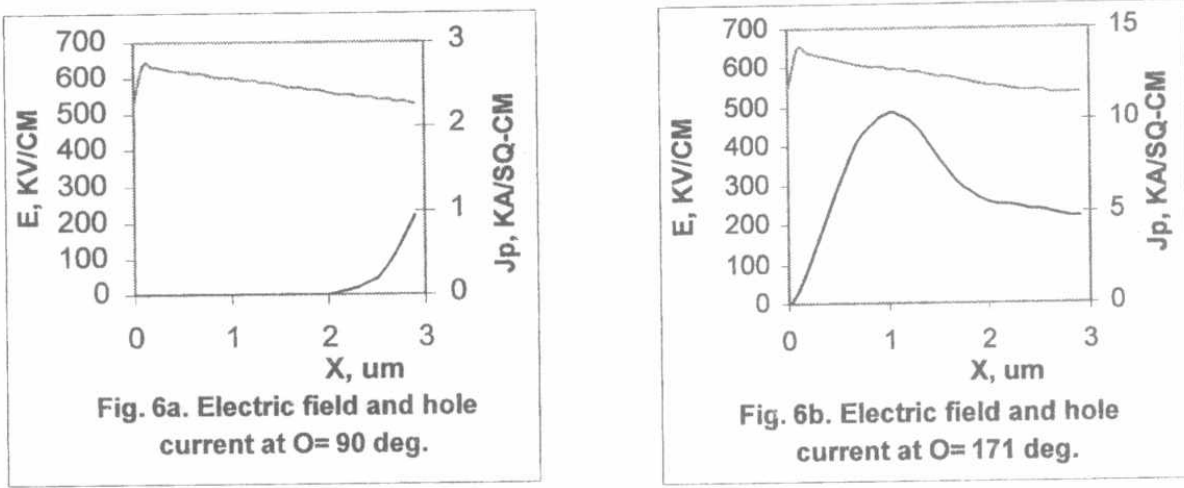

Fig. 6b. Electric field and hole current at $O=171 \mathrm{deg}$.
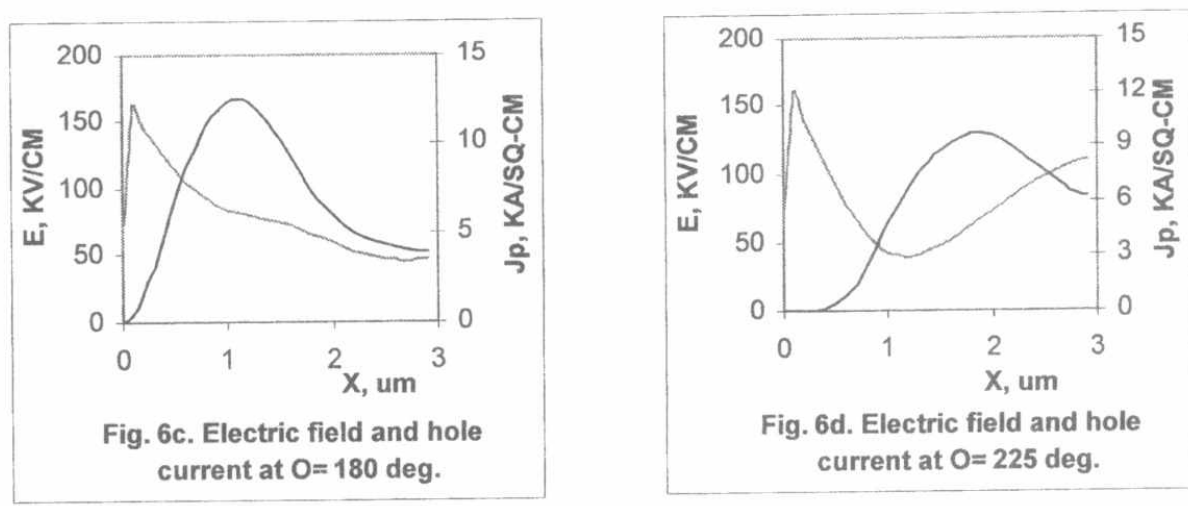

Fig. 6d. Electric field and hole current at $O=225 \mathrm{deg}$.

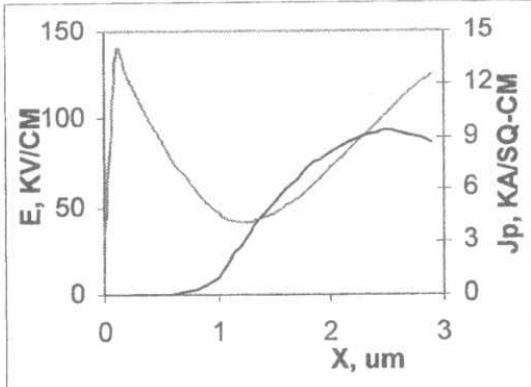

Fig. 6e. Electric field and hole current at $\mathrm{O}=\mathbf{2 7 0} \mathrm{deg}$.

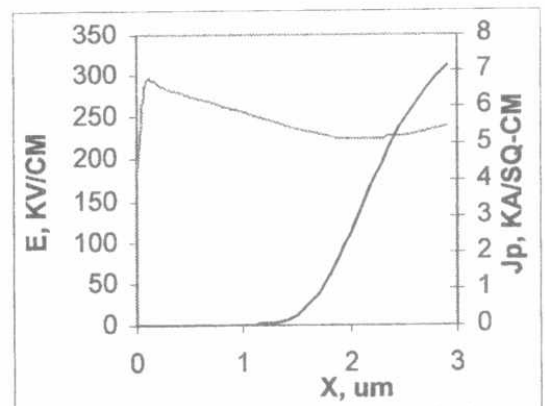

Fig. 6f. Electric field and hole current at $\mathrm{O}=360 \mathrm{deg}$. 


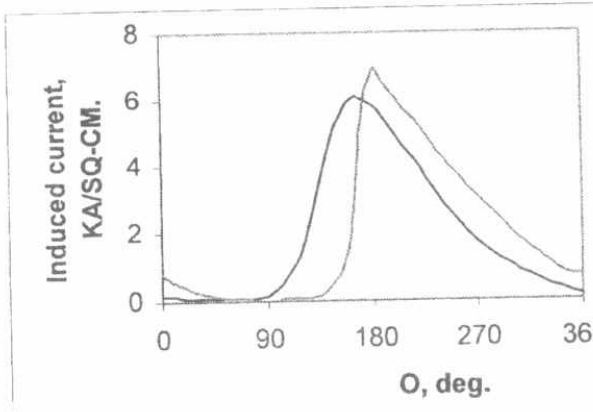

Fig. 7a. Induced current for VF and VC at Jdc $=2 \mathrm{KA} / \mathrm{SQ}-\mathrm{CM}$.
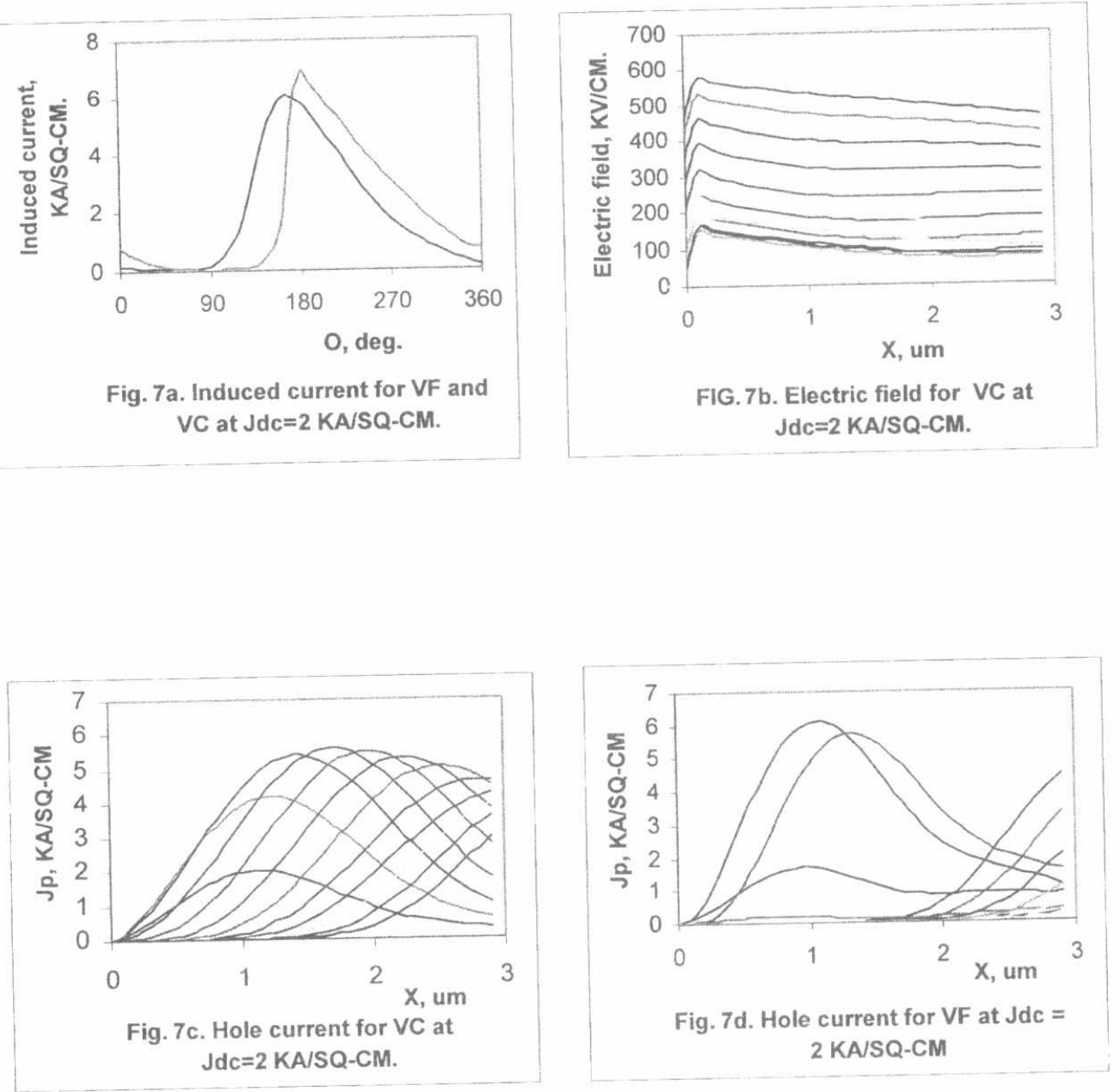